\title{
Evolution of Entanglement Entropy in One-Dimensional Systems
}

\author{
Pasquale Calabrese ${ }^{1}$ and John Cardy ${ }^{1,2}$ \\ ${ }^{1}$ Rudolf Peierls Centre for Theoretical Physics, 1 Keble Road, Oxford OX1 3NP, U.K.* \\ ${ }^{2}$ All Souls College, Oxford.
}

\begin{abstract}
We study the unitary time evolution of the entropy of entanglement of a one-dimensional system between the degrees of freedom in an interval of length $\ell$ and its complement, starting from a pure state which is not an eigenstate of the hamiltonian. We use path integral methods of quantum field theory as well as explicit computations for the transverse Ising spin chain. In both cases, there is a maximum speed $v$ of propagation of signals. In general the entanglement entropy increases linearly with time $t$ up to $t=\ell / 2 v$, after which it saturates at a value proportional to $\ell$, the coefficient depending on the initial state. This behavior may be understood as a consequence of causality.
\end{abstract}

*Address for correspondence 


\section{INTRODUCTION}

Understanding the degree of entanglement of systems with many degrees of freedom, such as quantum spin chains, is currently one of the most challenging problems connecting quantum information science and statistical physics. In the last few years several measures of entanglement have been proposed [1,2], and have been calculated (analytically in the simplest instances, otherwise numerically) in the ground state of several many-body systems. Despite a wide investigation, there is still no consensus on the correct measure of entanglement in the ground state of a many-body system. In this paper we will consider only the entanglement entropy, since it is the measure most readily suited to analytic investigations, especially if quantum field theory methods are employed [3].

The entanglement entropy is defined as follows. Suppose the whole system is in a pure quantum state $|\Psi\rangle$, with density matrix $\rho=|\Psi\rangle\langle\Psi|$, and an observer A measures only a subset $A$ of a complete set of commuting observables, while another observer B may measure the remainder. A's reduced density matrix is $\rho_{A}=\operatorname{Tr}_{B} \rho$. The entanglement entropy is just the von Neumann entropy $S_{A}=-\operatorname{Tr}_{A} \rho_{A} \log \rho_{A}$ associated with this reduced density matrix. It is easy to check that $S_{A}=S_{B}$. For an unentangled product state, $S_{A}=0$. Conversely, $S_{A}$ should be a maximum for a maximally entangled state. One of the most striking features of the entanglement entropy is the universal behavior displayed at and close to a quantum phase transition. In fact, close to a quantum critical point, where the correlation length $\xi$ is much larger than the lattice spacing $a$, the long-distance behavior of the correlations in the ground state of a quantum spin chain are effectively described by a $1+1$ dimensional quantum field theory. At the critical point, where $\xi$ diverges, the field theory, if Lorentz-invariant, is also a conformal field theory (CFT). In the latter case, it has been shown that, if $A$ is an interval of length $\ell$ in an infinite chain, $S_{A} \sim(c / 3) \log \ell[4,3]$, where $c$ is the central charge of the corresponding CFT. In higher dimensions, the critical entanglement entropy $S_{A}$ scales like the area separating $A$ and $B[5,2,3]$. These results have been verified by analytic and numerical calculations on integrable quantum spin chains and in more complicated systems $[2,6-10]$. When the correlation length $\xi$ is large, but finite, it has been shown that for 1-dimensional systems $S_{A}=\mathcal{A}(c / 6) \log \xi[3]$, where $\mathcal{A}$ is the number of boundary points between $A$ and $B$ (the 1-d "area"). This has been confirmed in some integrable models with $\mathcal{A}=1[3]$ and $\mathcal{A}=2[7,8]$. Finally the dependence of the entanglement entropy on the central charge has been the starting point for understanding the connection between irreversibility along renormalization group trajectories (Zamolodchikov's $c$-theorem [11]) and loss of entanglement [2,12].

A less investigated situation is how (one measure of) entanglement evolves when the system is prepared in a state that is not an eigenstate of the corresponding hamiltonian. Only a few works considered some entanglement measures in rather specific models $[13,10]$, thus universal features as in the ground state have not yet emerged. In this paper we mainly consider a scenario that is simply realized experimentally, for example in ultracold atoms [14,15]. Consider a quantum system at $T=0$ with an hamiltonian $H(\lambda)$ depending on a tunable experimental parameter $\lambda$ (for simplicity one may think of the Ising chain in a transverse magnetic field $\lambda[16]$, but the scenario is more general). The system is prepared in a pure state $\left|\psi_{0}\right\rangle$, which corresponds to an eigenstate of $H\left(\lambda_{0}\right)$ with $\lambda_{0} \neq \lambda$. Then, for example, at time $t=0$ the parameter is suddenly quenched from $\lambda_{0}$ to $\lambda$. In general we 
expect that $\left|\psi_{0}\right\rangle$ is not an eigenstate of $H(\lambda)$, and thus the system evolves unitarily according to the equations of motion given by $H(\lambda)$, in the absence of any dissipation (as we assume throughout the paper). In general, the system does not relax to its ground state, as it is the case in the presence of dissipation. Within this scenario we consider the time-evolution of entanglement entropy.

In this paper we concentrate on the case of one space dimension, using two complementary methods which have been successfully applied to the time-independent case. The first uses the path integral representation and the methods of quantum field theory. It is straightforward formally to write a path integral representation for the reduced density matrix

$$
\rho_{A}(t)=\operatorname{Tr}_{B} e^{-i H(\lambda) t}\left|\psi_{0}\right\rangle\left\langle\psi_{0}\right| e^{i H(\lambda) t}
$$

and then, following [3], to write the moments $\operatorname{Tr}_{A} \rho_{A}^{n}(t)$ as path integrals over an $n$-sheeted surface with branch cuts along A. To proceed further however, we assume that $H(\lambda)$ corresponds to a continuum conformal field theory. As in any continuum field theory, the energy fluctuations in the state $\left|\psi_{0}\right\rangle$ are then divergent. The simplest way of regularizing these while keeping the calculation tractable is to modify the state according to the prescription $\left|\psi_{0}\right\rangle \rightarrow e^{-\epsilon H(\lambda)}\left|\psi_{0}\right\rangle$, which filters out the high energy components.

With this modification, we find that when $A$ is an interval of length $\ell$, in the limit when $\ell / \epsilon, t / \epsilon \gg 1$, the entanglement entropy $S_{A}$ grows linearly with $t$, up to a time $t \sim \ell / 2$ (in units where the speed of the elementary excitations is unity). Thereafter it saturates at a value $S_{A} \sim \pi c \ell / 12 \epsilon$. These results, and the crossover time $t \sim \ell / 2$, can be understood in terms of causality applied to entangled left- and right-moving pairs of quasiparticles emitted from the initial state.

As a complement to the general conformal field theory calculations, we calculate the time-dependent entanglement entropy $S_{A}$ for the simplest integrable model: The Ising chain in a transverse magnetic field. The reason for this calculation is twofold. On the one hand, it is a practical check of the CFT results just summarized and can help understanding problems connected with the regularization $\epsilon$. On the other, it allows exact calculations even in the non-critical (massive) phase, thus generalizing the CFT results.

We consider the time evolution from the ground state at a given transverse magnetic field $h_{0}$, according to the equation of motion given by $H(h)$ with $h \neq h_{0}$. We obtain the remarkable analytic results (equation (3.19)) that for $t \rightarrow \infty, S_{A}$ is proportional to $\ell$ for each pair of $h$ and $h_{0}$ (as long as $h \neq h_{0}$ ). This confirms the CFT results and further shows that the linear dependence on $\ell$ for large times is not a peculiarity of the critical evolution. Thus for each given $h$, there is an arbitrary large entanglement entropy in the asymptotic state, contrarily to the ground-state case, where infinite entropy is allowed only at criticality. Furthermore we obtain that $\lim _{\ell \rightarrow \infty} S_{A} / \ell$ is bounded from above by $2 \log 2-1$ (the value found for $h_{0}=\infty, h=1$ ). This maximum value of the entanglement entropy is less than $\ell \log 2$, a rigorous bound for a generic state of $\ell$ spins one-half. An interesting (and not understood) feature of the leading term of $S_{A}$ for $t \gg \ell \rightarrow \infty$ is that it is invariant under the exchange $h \leftrightarrow h_{0}$.

For finite times we find a linear increase of $S_{A}$ with $t$, for all $h \neq h_{0}$, not only at criticality. The crossover between the linear and non-linear regime happens always at $t^{*}=\ell / 2$, for large $\ell$. However for $t>\ell / 2, S_{A}$ does not immediately saturate to the asymptotic value, but is 
a slowly increasing function of the time. In Sec. IV, we ascribe this difference between lattice and CFT results to the non-linear dispersion relation of quasiparticles emitted from the initial state. When both $h$ and $h_{0}$ get closer to the critical field, the time dependence becomes sharper and more similar to the CFT results.

The layout of this paper is as follows. In the next section, we discuss the time dependence on the entanglement entropy within CFT, and in Sec. III we consider the quantum Ising model in a transverse field (some cumbersome details of the calculation are postponed to two appendices). In Sec. IV we show that the results obtained may be explained in terms of a physical picture invoking causality. Finally in Sec. V we discuss some points that need further investigation.

\section{TIME EVOLUTION OF ENTANGLEMENT ENTROPY IN QUANTUM FIELD THEORY}

\section{A. Path integral formulation}

Suppose (in the notation of [3]) we prepare the system in a state $\left|\psi_{0}(x)\right\rangle$ and unitarily evolve it with the hamiltonian $H$. The matrix elements of the density matrix at time $t$ are

$$
\left\langle\psi^{\prime \prime}\left(x^{\prime \prime}\right)|\rho(t)| \psi^{\prime}\left(x^{\prime}\right)\right\rangle=Z_{1}^{-1}\left\langle\psi^{\prime \prime}\left(x^{\prime \prime}\right)\left|e^{-i t H-\epsilon H}\right| \psi_{0}(x)\right\rangle\left\langle\psi_{0}(x)\left|e^{+i t H-\epsilon H}\right| \psi^{\prime}\left(x^{\prime}\right)\right\rangle .
$$

We have included damping factors $e^{-\epsilon H}$ in such a way as to make the path integral absolutely convergent. We shall see at the end of the calculation whether it is justified to remove them.

Each of the factors may be represented by an analytically continued path integral in imaginary time: the first one over fields $\psi(x, \tau)$ which take the boundary values $\psi_{0}(x)$ on $\tau=-\epsilon-i t$ and $\psi^{\prime \prime}(x)$ on $\tau=0$, and the second with $\psi(z, \tau)$ taking the values $\psi^{\prime}(x)$ on $\tau=0$ and $\psi_{0}(x)$ on $\tau=\epsilon-i t$. This is illustrated in Fig. 1.

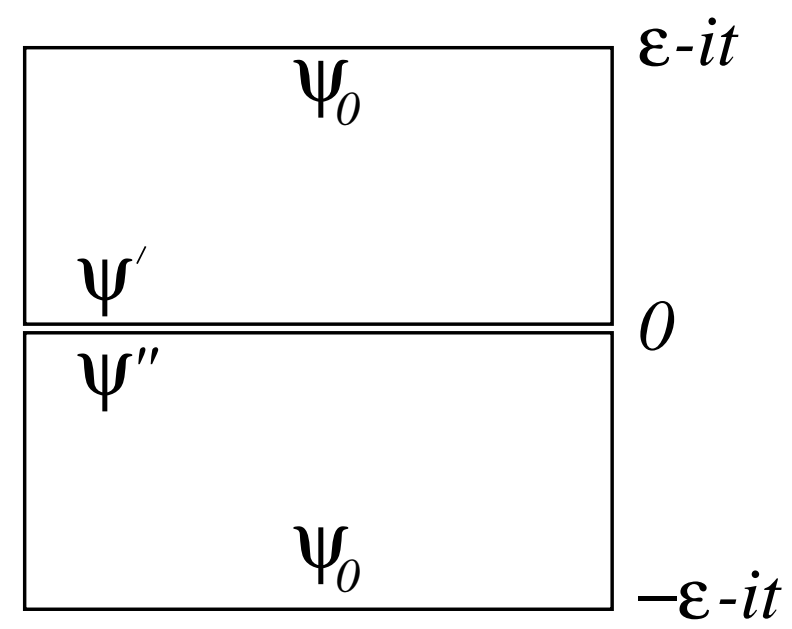

FIG. 1. Space-imaginary time regions for the density matrix in (2.1)

The normalization factor

$$
Z_{1}=\left\langle\psi_{0}(x)\left|e^{-2 \epsilon H}\right| \psi_{0}(x)\right\rangle
$$


which guarantees that $\operatorname{Tr} \rho=1$ is given by the euclidean path integral over imaginary time $2 \epsilon$, with initial and final conditions both equal to $\psi_{0}(x)$. This is the same as sewing together the two edges in Fig. 1 along $\tau=0$.

As in [3], the reduced density matrix $\rho_{A}(t)$ is giving by sewing together along $\tau=0$ only those parts of the $x$-axis corresponding to points in $\mathrm{B}$, leaving open slits along $\mathrm{A}$, and $\operatorname{Tr} \rho_{A}^{n}$ is given by sewing together $n$ copies of this in a cyclic fashion. Thus

$$
\operatorname{Tr} \rho_{A}^{n}=Z_{n} / Z_{1}^{n}
$$

where $Z_{n}$ is the path integral on an $n$-sheeted surface, where the edges of each sheet correspond to imaginary times $-\tau_{1}$ and $\tau_{2}$, analytically continued to $\tau_{1}=\epsilon+i t$ and $\tau_{2}=\epsilon-i t$, and the branch points lie along $\tau=0$ at the boundaries points between A and B. Finally, as derived in [3], the entanglement entropy is given by

$$
S_{A}=-\left.\frac{\partial}{\partial n} \operatorname{Tr} \rho_{A}^{n}\right|_{n=1}
$$

\section{B. Conformal mapping}

Now consider the case when $H$ is critical and the field theory is a CFT. Under the renormalization group any translationally invariant boundary condition is supposed to flow into a boundary fixed point, satisfying conformal boundary conditions. Thus we may assume that the state $\left|\psi_{0}\right\rangle$ corresponds to such boundary conditions on sufficiently long length scales. However this means that the conclusions will be valid only asymptotically.

For real $\tau$ the strip geometry described above may be obtained from the upper halfplane by the conformal mapping $w=(2 \epsilon / \pi) \log z$, with the images of the slits lying along $\arg z=\pi \tau_{1} / 2 \epsilon$. The result for $Z_{n} / Z_{1}^{n}$ in the upper half $z$-plane, for an arbitrary number of branch points, was given in [3]. It has the form of a correlation function of local operators $\Phi_{ \pm n}$ located at the branch points, which transforms simply under conformal mappings.

First consider the case when $\mathrm{A}$ is a slit of length $\ell$ (and $\mathrm{B}$ is the rest of the real axis.) In that case we can take the image of the branch points to be $z_{1}=\rho^{-1} e^{i \pi \tau_{1} / 2 \epsilon}$ and $z_{2}=\rho e^{i \pi \tau_{1} / 2 \epsilon}$ where $\rho=e^{\pi \ell / 4 \epsilon}$. From [3] in the $z$-plane we have

$$
\operatorname{Tr} \rho_{A}^{n}=\left\langle\Phi_{n} \Phi_{-n}\right\rangle \sim c_{n}\left(\frac{\left|z_{1}-\bar{z}_{2}\right|\left|z_{2}-\bar{z}_{1}\right|}{\left|z_{1}-z_{2}\right|\left|\bar{z}_{1}-\bar{z}_{2}\right|\left|z_{1}-\bar{z}_{1}\right|\left|z_{2}-\bar{z}_{2}\right|}\right)^{2 n \Delta_{n}}
$$

where $\Delta_{n}=(c / 24)\left(1-1 / n^{2}\right)$ in the notation of [3]. This formula is supposed to be valid in the euclidean region, when all the separations are large. Under the conformal mapping,

$$
\left\langle\Phi_{n}\left(w_{1}\right) \Phi_{-n}\left(w_{2}\right)\right\rangle=\left|w^{\prime}\left(z_{1}\right) w^{\prime}\left(z_{2}\right)\right|^{-2 n \Delta_{n}}\left\langle\Phi_{n}\left(z_{1}\right) \Phi_{-n}\left(z_{2}\right)\right\rangle .
$$

After some algebra and continuing to $\tau_{1}=\epsilon+i t$, we find

$$
\operatorname{Tr} \rho_{A}^{n}(t) \sim c_{n}(\pi / 2 \epsilon)^{4 n \Delta_{n}}\left(\frac{e^{\pi \ell / 2 \epsilon}+e^{-\pi \ell / 2 \epsilon}+2 \cosh (\pi t / \epsilon)}{\left(e^{\pi \ell / 4 \epsilon}-e^{-\pi \ell / 4 \epsilon}\right)^{2} \cosh ^{2}(\pi t / 2 \epsilon)}\right)^{2 n \Delta_{n}} .
$$


In the case where $\ell / \epsilon$ and $t / \epsilon$ are large this simplifies to

$$
c_{n}(\pi / 2 \epsilon)^{4 n \Delta_{n}}\left(\frac{e^{\pi \ell / 2 \epsilon}+e^{\pi t / \epsilon}}{e^{\pi \ell / 2 \epsilon} \cdot e^{\pi t / \epsilon}}\right)^{2 n \Delta_{n}} .
$$

Differentiating wrt $n$ to get the entropy,

$$
S_{A}(t) \sim \begin{cases}\frac{\pi c t}{6 \epsilon} & (t<\ell / 2), \\ \frac{\pi c \ell}{12 \epsilon} & (t>\ell / 2),\end{cases}
$$

that is $S_{A}(t)$ increases linearly until it saturates at $t=\ell / 2$. The sharp cusp in this asymptotic result is rounded over a region $|t-\ell / 2| \sim \epsilon$.

However we see that $\epsilon$ enters in an essential way. The reason is that, in a continuum field theory (as compared with a quantum spin model) a state like $\left|\psi_{0}\right\rangle$ has infinitely large mean energy (as well as divergent energy fluctuations). In order to make sense of the result it is necessary to filter out the high-energy components of the state. Within the path integral approach, this is most easily enforced with a cut-off function $e^{-\epsilon E}$. To compare with results from a lattice spin model we should presumably take $\epsilon$ to be of the order of the lattice spacing. The linear behavior in $t$ for $t<\ell / 2$, the break at $t=\ell / 2$, and the saturation at a value $\propto \ell$ all agree with our exact results for the transverse Ising spin chain, in Sec. III, although there are other differences in detail.

\section{DYNAMICS OF ENTANGLEMENT ENTROPY IN THE QUANTUM ISING CHAIN}

As a complement to the general CFT calculation just presented, in this section we describe how analogous results can be found in an analytically tractable model. We consider the Ising spin chain in a transverse magnetic field, which has a quantum phase transition between a ferromagnetic and a (quantum) paramagnetic phase.

The model is defined by the hamiltonian

$$
H_{I}(h)=-\frac{1}{2} \sum_{j}\left[\sigma_{j}^{x} \sigma_{j+1}^{x}+h \sigma_{j}^{z}\right],
$$

where $\sigma_{j}^{x, z}$ are the Pauli matrix acting on the spin at the site $j$ of an infinite chain. The quantum critical point is located at $h=1[16]$. We consider the time evolution from an initial state $\left|\psi_{0}\right\rangle$ that is an eigenstate of $H_{I}$ for a field $h_{0} \neq h$. This experimentally means quenching at $t=0$ the magnetic field from $h_{0}$ to $h$. We consider only the case $h, h_{0} \geq 1$. The generalizations to the case $h, h_{0}<1$ and to more general spin chains, such as the XY model [16] are straightforward and we will not consider them here.

The determination of the time-dependent state $|\psi(t)\rangle=e^{-i H_{I}(h) t}\left|\psi_{0}\right\rangle$ (and consequently of the entanglement entropy) proceeds with the Jordan-Wigner transformation in terms of 
Dirac or Majorana fermionic operators. All the details of these computations can be found in the Appendix A.

The final result is that the time-dependent entanglement entropy for $\ell$ consecutive spins in the chain can be obtained (analogously to the ground state case [2]) from the correlation matrix of the Majorana operators

$$
\check{a}_{2 l-1} \equiv\left(\prod_{m<l} \sigma_{m}^{z}\right) \sigma_{l}^{x}, \quad \check{a}_{2 l} \equiv\left(\prod_{m<l} \sigma_{m}^{z}\right) \sigma_{l}^{y} .
$$

We introduce the matrix $\Gamma_{\ell}^{A}$ through the relation $\left\langle\check{a}_{m} \check{a}_{n}\right\rangle=\delta_{m n}+i \Gamma_{\ell m n}^{A}$ with $1 \leq m, n \leq \ell$. It has the form of a block Toeplitz matrix

$$
\Gamma_{\ell}^{A}=\left[\begin{array}{cccc}
\Pi_{0} & \Pi_{-1} & \cdots & \Pi_{1-\ell} \\
\Pi_{1} & \Pi_{0} & & \vdots \\
\vdots & & \ddots & \vdots \\
\Pi_{\ell-1} & \cdots & \cdots & \Pi_{0}
\end{array}\right], \quad \Pi_{l}=\left[\begin{array}{cc}
-f_{l} & g_{l} \\
-g_{-l} & f_{l}
\end{array}\right]
$$

with

$$
\begin{aligned}
g_{l} & =\frac{1}{2 \pi} \int_{0}^{2 \pi} d \varphi e^{-i \varphi l} e^{-i \theta_{\varphi}}\left(\cos \Phi_{\varphi}-i \sin \Phi_{\varphi} \cos 2 \epsilon_{\varphi} t\right), \\
f_{l} & =\frac{i}{2 \pi} \int_{0}^{2 \pi} d \varphi e^{-i \varphi l} \sin \Phi_{\varphi} \sin 2 \epsilon_{\varphi} t
\end{aligned}
$$

where

$$
\begin{aligned}
\epsilon_{\varphi} & =\sqrt{(h-\cos \varphi)^{2}+\sin ^{2} \varphi} \\
\epsilon_{\varphi}^{0} & =\sqrt{\left(h_{0}-\cos \varphi\right)^{2}+\sin ^{2} \varphi} \\
e^{-i \theta_{\varphi}} & =\frac{\cos \varphi-h-i \sin \varphi}{\epsilon_{\varphi}}, \\
\sin \Phi_{\varphi} & =\frac{\sin \varphi\left(h_{0}-h\right)}{\epsilon_{\varphi} \epsilon_{\varphi}^{0}} \\
\cos \Phi_{\varphi} & =\frac{1-\cos \varphi\left(h+h_{0}\right)+h h_{0}}{\epsilon_{\varphi} \epsilon_{\varphi}^{0}} .
\end{aligned}
$$

Calling the eigenvalues of $\Gamma_{\ell}^{A}$ as $\pm i \nu_{m}, m=1 \ldots \ell$, the entanglement entropy is $S=$ $\sum_{m=1}^{\ell} H\left(\nu_{m}\right)$ where $H(x)$ is

$$
H(x)=-\frac{1+x}{2} \log \frac{1+x}{2}-\frac{1-x}{2} \log \frac{1-x}{2} .
$$

The derivation of all these results is in the Appendix A.

Note that the first trivial result is that for $t=0$ and for $h=h_{0}$, the correlation matrix reduces to that of the ground state with field $h_{0}$, as it should. 


\section{A. Analytical results for $t \rightarrow \infty$}

In the limit $t \rightarrow \infty$ the matrix $\Gamma_{\ell}^{A}$ simplifies in such a way that analytical calculations are feasible for all $h, h_{0}$. In fact, for $t \rightarrow \infty$, the time-dependent sine and cosine appearing in Eqs. (3.4) average to zero, and $\Gamma_{\ell}^{A}$ has a simpler form with $f_{l}=0$ and

$$
g_{l}=\frac{1}{2 \pi} \int_{0}^{2 \pi} d \varphi e^{-i \varphi l} \frac{e^{-i \varphi}-h}{\epsilon_{\varphi}^{2} \epsilon_{\varphi}^{0}}\left(1-\cos \varphi\left(h+h_{0}\right)+h h_{0}\right) .
$$

Thus it can be written as a block-Toeplitz matrix with

$$
\Pi_{l}=\frac{1}{2 \pi} \int_{0}^{2 \pi} d \varphi e^{-i \varphi l} \Pi(\varphi)
$$

and

$$
\Pi(\varphi)=\left[\begin{array}{cc}
0 & \frac{e^{-i \varphi}-h}{\epsilon_{\varphi}^{2}} \frac{1-\cos \varphi\left(h+h_{0}\right)+h h_{0}}{e_{\varphi}^{0}} \\
-\frac{e^{i \varphi}-h}{\epsilon_{\varphi}^{2}} \frac{1-\cos \varphi\left(h+h_{0}\right)+h h_{0}}{e_{\varphi}^{0}} & 0
\end{array}\right] .
$$

In this case, following the method used for the ground state in Refs. [6,7] it is possible to calculate the leading behavior of $S_{\ell}(\infty)$ for large $\ell$. Let us introduce $[6,7]$

$$
\begin{aligned}
\tilde{\Gamma}_{\ell} & =i \lambda I_{\ell}-\Gamma_{\ell}^{A}, \\
\tilde{\Pi}(\varphi) & =i \lambda I_{1}-\Pi(\varphi), \\
e(y, x) & =-\frac{y+x}{2} \log \frac{y+x}{2}-\frac{y-x}{2} \log \frac{y-x}{2},
\end{aligned}
$$

where $I_{\ell}$ is the $2 \ell \times 2 \ell$ identity matrix.

The determinant of $\tilde{\Gamma}_{\ell}$ is

$$
D_{\ell}(\lambda) \equiv \operatorname{det} \tilde{\Gamma}_{\ell}=(-1)^{\ell} \prod_{j}^{\ell}\left(\lambda^{2}-\nu_{j}^{2}\right),
$$

so we can use Cauchy theorem to rewrite the entropy as [7]

$$
S_{\ell}=\lim _{\epsilon \rightarrow 0^{+}} \frac{1}{4 \pi i} \oint_{C} d \lambda e(1+\epsilon, \lambda) \frac{d}{d \lambda} \ln D_{\ell}(\lambda),
$$

where the contour $C$ depends on the parameter $\epsilon$ and includes the interval $[-1,1]$, so to encircle all the zeros of $D_{\ell}(\lambda)$. This guarantees that the branch points of $e(1+\epsilon, \lambda)$ are outside $C$ and $e(1+\epsilon, \lambda)$ is analytic there. As $\epsilon$ goes to zero the contour approaches the interval $[-1,1]$.

An old short overview about theorems concerning this type of matrices can be found in [17]. In particular, it is known that the large $\ell$ asymptotic behavior of the determinant of 
(a)

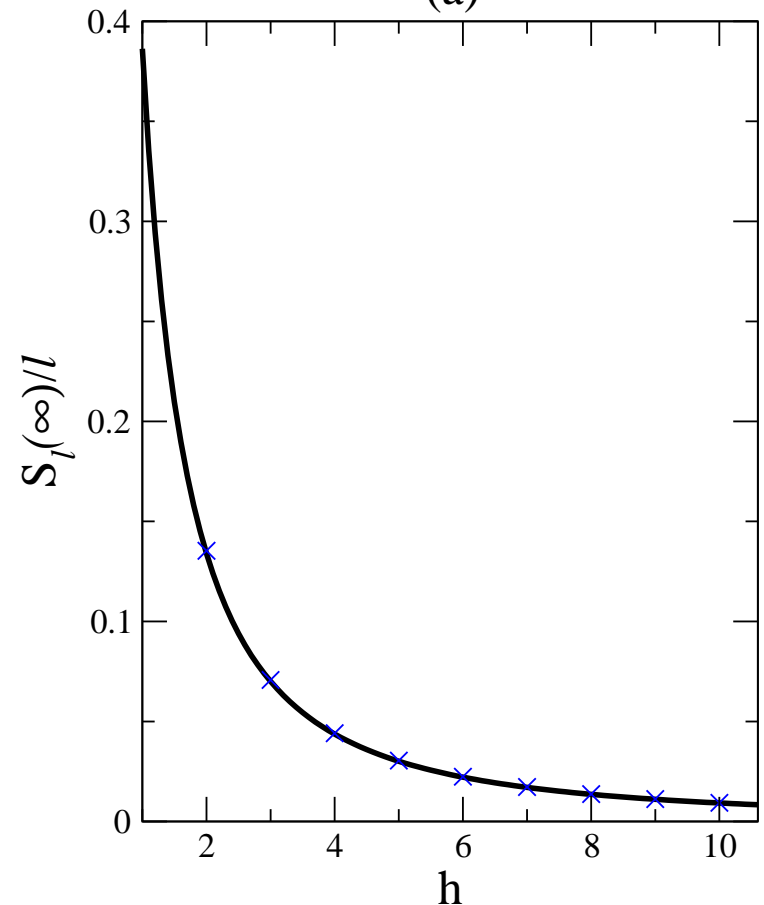

(b)

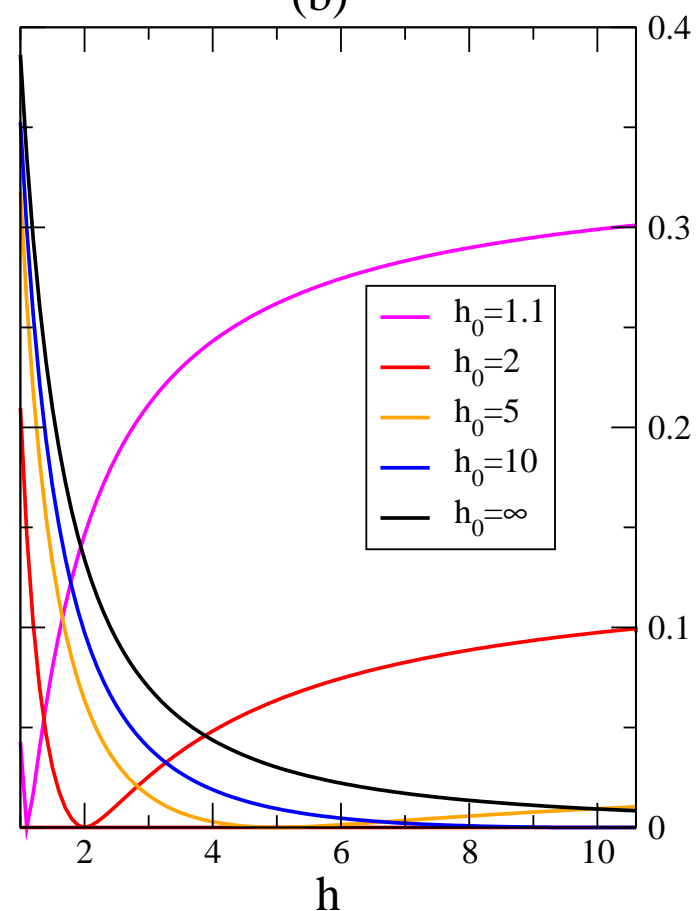

FIG. 2. Leading asymptotic entanglement entropy for unit length of the interval, as function of $h$ Eq. (3.19). (a) The case $h_{0}=\infty$ (full line) is compared with the numerical results (crosses) obtained by diagonalizing $\Gamma_{\ell}^{A}$ for $\ell=200$ and using the exact coefficients Eq. (B1). (b) As in (a), but for several finite values of $h_{0}$.

a block Toeplitz matrix $T_{\ell}$ (under some hypothesis satisfied in our case $t=\infty$ ) is given by the generalization of the Szego lemma [17]

$$
\log \operatorname{det} T_{\ell}=\ell \frac{1}{2 \pi} \int_{0}^{2 \pi} d \varphi \log [\operatorname{det} a(\varphi)]+O(\log \ell)
$$

where $a(\varphi)$ is the generating matrix-function of $T_{\ell}$ (in our case $\tilde{\Pi}(\varphi)$ ). Thus

$$
\log \operatorname{det} \tilde{\Gamma}_{\ell}=\frac{\ell}{2 \pi} \int_{0}^{2 \pi} d \varphi \log [\operatorname{det} \tilde{\Pi}(\varphi)]+O(\log \ell),
$$

so (we omit $O(\log \ell))$

$$
\frac{d \log \operatorname{det} \tilde{\Gamma}_{\ell}(\lambda)}{d \lambda}=\frac{\ell}{2 \pi} \int_{0}^{2 \pi} d \varphi \frac{2 \lambda}{\lambda^{2}-\frac{\left(1-\cos \varphi\left(h+h_{0}\right)+h h_{0}\right)^{2}}{\left(h^{2}+1-2 h \cos \varphi\right)\left(h_{0}^{2}+1-2 h_{0} \cos \varphi\right)}} .
$$

Inserting this expression in Eq. (3.14) we get

$$
\begin{aligned}
S_{\ell}(\infty) & =\lim _{\epsilon \rightarrow 0^{+}} \frac{1}{4 \pi i} \oint_{C} d \lambda e(1+\epsilon, \lambda) \frac{d}{d \lambda} \ln \operatorname{det} \tilde{\Gamma}_{\ell}(\lambda)= \\
& =\lim _{\epsilon \rightarrow 0^{+}} \frac{1}{4 \pi i} \oint_{C} d \lambda e(1+\epsilon, \lambda) \frac{\ell}{2 \pi} \int_{0}^{2 \pi} d \varphi \frac{2 \lambda}{\lambda^{2}-\frac{\left(1-\cos \varphi\left(h+h_{0}\right)+h h_{0}\right)^{2}}{\left(h^{2}+1-2 h \cos \varphi\right)\left(h_{0}^{2}+1-2 h_{0} \cos \varphi\right)}} .
\end{aligned}
$$


Changing the order of the integrals, using the residue theorem, and the property $e(y, x)=$ $e(y,-x)$ we get $^{1}$

$$
\begin{aligned}
S_{\ell}(\infty) & =\lim _{\epsilon \rightarrow 0^{+}} \frac{\ell}{2 \pi} \frac{1}{4 \pi i} \int_{0}^{2 \pi} d \varphi \oint_{C} d \lambda e(1+\epsilon, \lambda) \frac{2 \lambda}{\lambda^{2}-\frac{\left(1-\cos \varphi\left(h+h_{0}\right)+h h_{0}\right)^{2}}{\left(h^{2}+1-2 h \cos \varphi\right)\left(h_{0}^{2}+1-2 h_{0} \cos \varphi\right)}} \\
& =\frac{\ell}{2 \pi} \int_{0}^{2 \pi} d \varphi H\left(\frac{1-\cos \varphi\left(h+h_{0}\right)+h h_{0}}{\sqrt{\left(h^{2}+1-2 h \cos \varphi\right)\left(h_{0}^{2}+1-2 h_{0} \cos \varphi\right)}}\right) .
\end{aligned}
$$

Let us discuss this result. First, independently of $h$ and $h_{0}, S_{\ell}(\infty) / \ell$ is non-vanishing (as long as $h \neq h_{0}$ ). Thus the asymptotic entanglement entropy for the Ising chain is always linear in $\ell$, not only at the critical point as we already knew from CFT. For $h=1, h_{0}=\infty$ it reduces to $S_{\ell}(\infty) / \ell=2 \log 2-1$, as it can be understood from a very simple argument reported in Appendix B. This is the maximum value that $S_{\ell}(\infty) / \ell$ can asymptotically reach. In Fig. 2 (a) we depict $S_{\ell}(\infty) / \ell$ for a quench from $h_{0}=\infty$ as a function of $h$ : It is a monotonous decreasing function of $h$. In Fig. 2 (b) we depict $S_{\ell}(\infty) / \ell$ as function of $h$, for several $h_{0}$. For $h<h_{0}$ it is a decreasing function, while for $h>h_{0}$ it increases, and obviously it is zero at $h=h_{0}$. A further curious (and not understood) feature is that $S_{\ell}(\infty)$ is symmetric under the exchange of $h$ and $h_{0}$.

Note that in Eq. (3.19) corrections of the order $O(\log \ell)$ are neglected. These corrections are smaller than the leading term, but can be arbitrarily large in the limit $\ell \rightarrow \infty$. They are in principle obtainable using generalizations of the Fisher-Hartwig conjecture [7], and are not expected to be symmetric under $h \leftrightarrow h_{0}$.

\section{B. Some numerical results}

Now, we present the time-dependence of the entanglement entropy, as obtained by numerical diagonalization of $\Gamma_{\ell}^{A}$. We consider several quenches in the next subsections.

\section{Quench from $h_{0}=\infty$ to $h=1$.}

We start our numerical analysis from the case in which the system is quenched from a completely uncorrelated state $\left(h_{0}=\infty\right)$ to the critical point $(h=1)$. The time evolution is governed by the critical hamiltonian, so the results we obtain are expected to be welldescribed by CFT.

The numerical results, as obtained by diagonalizing numerically $\Gamma_{\ell}^{A}$, are shown in Fig. 3 for $\ell=20,40,60,100$. For all $\ell$ considered, $S_{\ell}(t)$ exhibits just two time-regimes: a linear

\footnotetext{
${ }^{1}$ In a similar way one can also calculate the the moments $\operatorname{Tr} \rho_{A}^{n}$. In fact, using the results reported in the Appendix, it is straightforward to show that $\log \operatorname{Tr} \rho_{A}^{n}$ is given by equation (3.19) with $H$ replaced by $H_{n}=\log \left\{[(1-x) / 2]^{n}+[(1+x) / 2]^{n}\right\}$. It follows that $\operatorname{Tr} \rho_{A}^{n} \propto e^{-f_{n}\left(h, h_{0}\right) \ell}$, where $f_{n}$ satisfies $f_{n}\left(h, h_{0}\right)=f_{n}\left(h_{0}, h\right)$.
} 


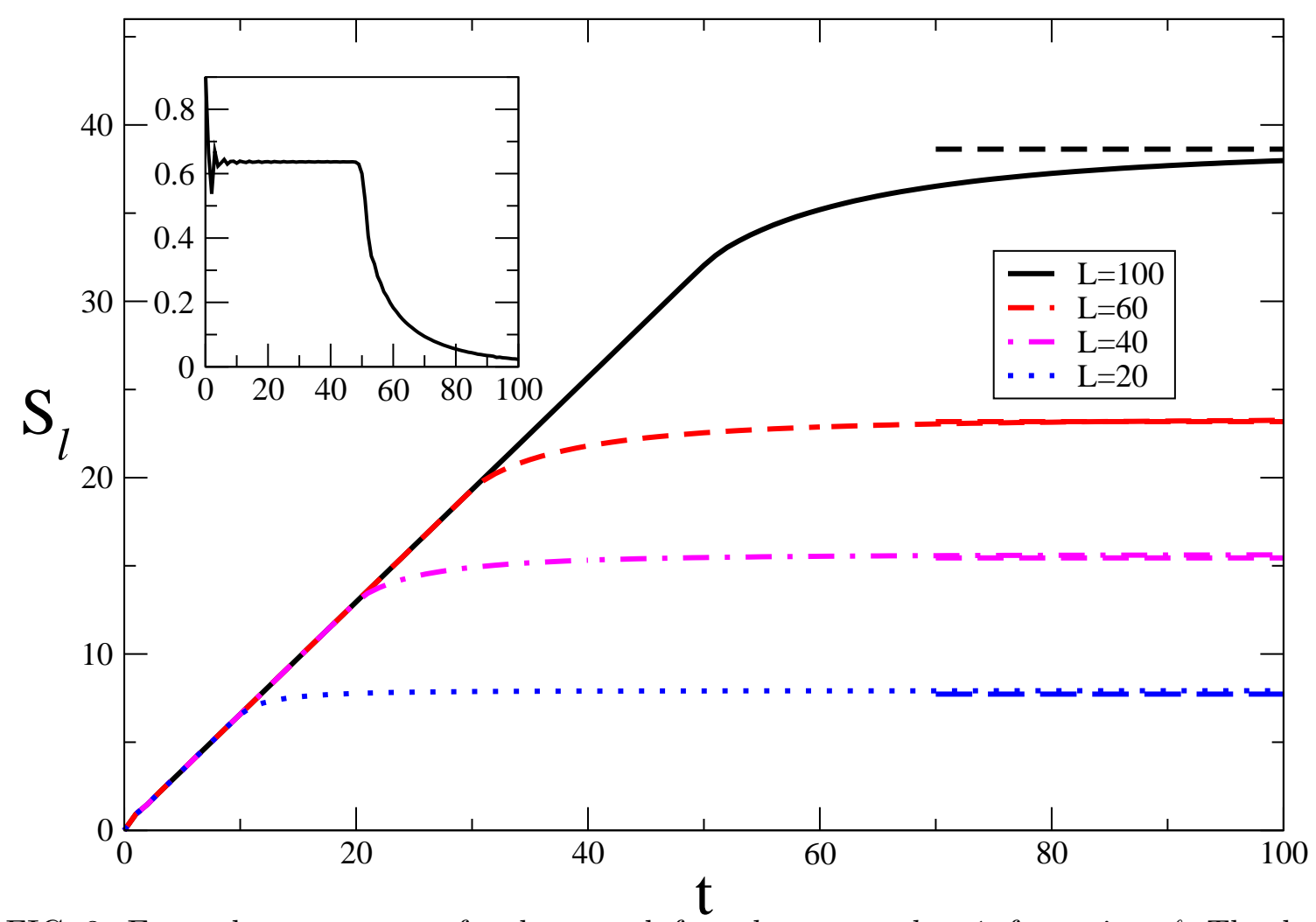

FIG. 3. Entanglement entropy for the quench from $h_{0}=\infty$ to $h=1$, for various $\ell$. The dashed lines are the leading asymptotic results for large $\ell$, cf. Eq. (3.19). The inset shows the derivative with respect to the time of $S_{100}(t)$.

one for $t<t^{*}=\ell / 2+O\left(\ell^{0}\right)$, and a nonlinear one for $t>t^{*}$. The crossover between the two regimes happens at $t^{*} \simeq \ell / 2$, in agreement with the CFT prediction. Note (inset of Fig. 3) that the derivative with respect to the time of $S_{100}(t)$ is practically constant for $t<50$, apart from an expected short-time non-universal behavior (that should disappear in the limit $\ell \rightarrow \infty)$. For $t<t^{*}$, all the $S_{\ell}(t)$ fall on the same master curve, independently of $\ell$. However, the entropy does not saturate to the asymptotic value exactly at $t^{*}$, as in CFT. In fact for large $t, S_{\ell}(t)$ is a slowly increasing function, even in the limit of large $\ell$.

The dashed lines in Fig. 3 are the leading asymptotic results for large $\ell$ as given by Eq. (3.19). The actual asymptotic value (obtained by numerical diagonalization) is always slightly larger than (3.19), showing that the first correction (most probably of the order $O(\log \ell))$ is positive.

\section{Quench from $h_{0}>1$ to $h=1$.}

To understand if the disagreement between CFT and the exact result for $t>t^{*}$ can be attributed simply to the particular initial condition $\left(h_{0}=\infty\right)$ or to deeper reasons, we consider now the case of a generic initial condition (i.e. finite $h_{0}$ ), always evolving according to the critical hamiltonian.

The numerical results for various $h_{0}$ (at fixed $\ell=60$ ) are displayed in Fig. 4. Even in this 


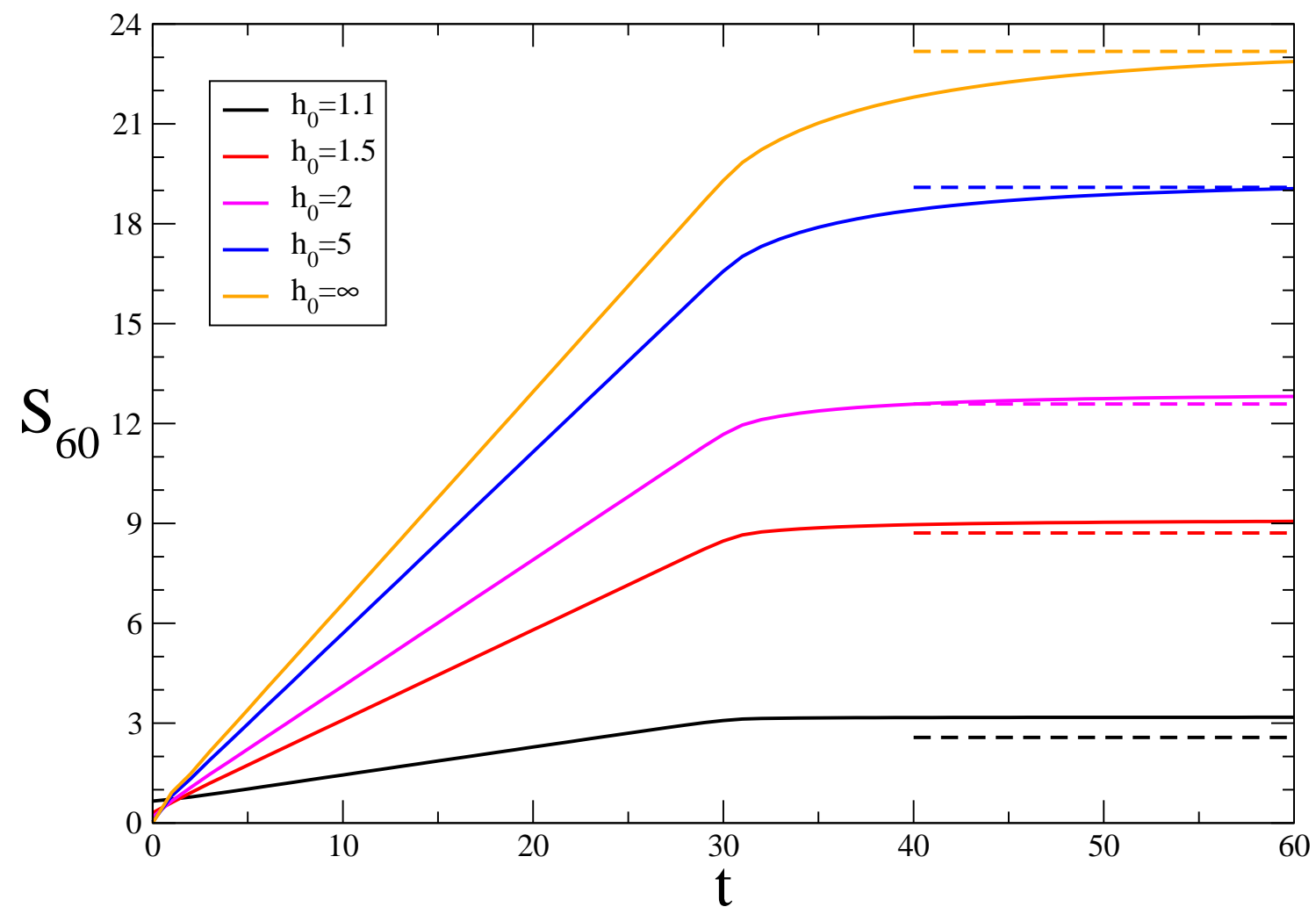

FIG. 4. $S_{60}(t)$ for the quench from $h_{0}=\infty, 5,2,1.5,1.1$ to $h=1$. The dashed lines are the leading asymptotic results for large $\ell$ Eq. (3.19).

case the curves show for $t<t^{*}$ a linear regime, in agreement with CFT. However, decreasing $h_{0}$ the time dependence for $t>t^{*}$ becomes sharper, i.e. $S_{\ell}(t)$ reaches the asymptotic value much faster than in the case $h_{0}=\infty$. Thus the curves look more similar to the CFT result.

In Fig. 4 the dashed lines are the leading asymptotic results for large $\ell$ as given by Eq. (3.19). Note that decreasing $h_{0}$, the positivity of the corrections to Eq. (3.19) is more and more apparent. In this case, it can be partially attributed to a non-vanishing value for $t=0$, that results in a finite shift of the entire curve.

\section{Quench from $h_{0}=\infty$ to $h>1$.}

In the lattice calculation we are performing we are not restricted to study the critical evolution. Thus, to understand how the entanglement entropy is affected by a non-critical evolution, we can simply diagonalize $\Gamma_{A}^{\ell}$ for $h \neq 1$. As a first case, we consider a quench from $h_{0}=\infty$ to a non critical value $h>1$. The numerical results, for fixed $\ell=100$, are shown in Fig. 5. The curves appear very similar to each other, and in particular to the critical case. There are two time regimes as in the critical case. The crossover from the linear to non-linear regime is always at $t^{*} \simeq \ell / 2$, independently of $\ell$ and $h$. A quite unexpected fact is that the all the curves fall on the same master curve, when rescaled according to $S_{100}(\infty)$ (see the inset in Fig. 5). We have no simple explanation for this. 


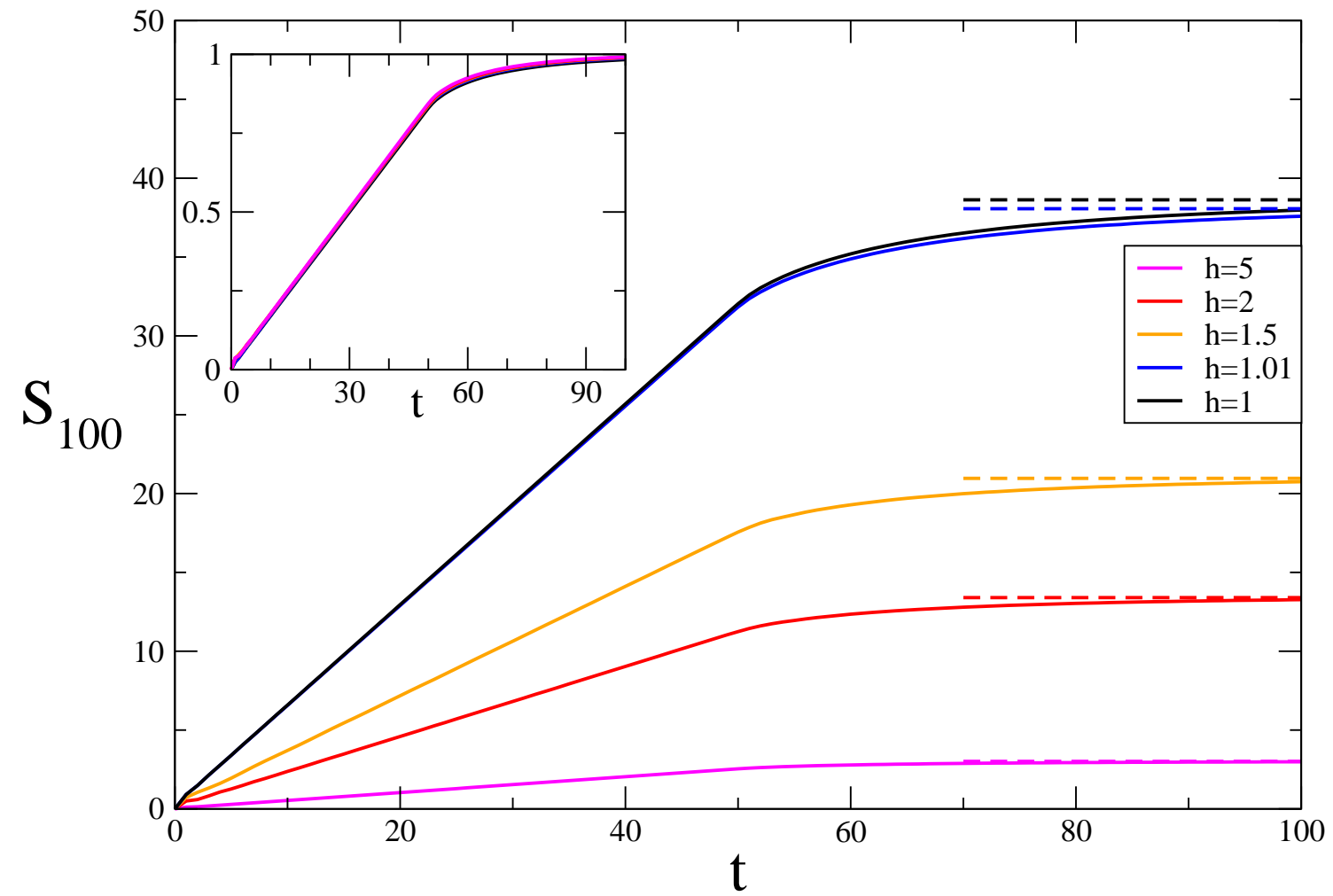

FIG. 5. $S_{100}(t)$ for the quench from $h_{0}=\infty$ to $h=5,2,1.5,1.01,1$. The dashed lines are the leading asymptotic results for large $\ell$ Eq. (3.19). The inset shows the rescaling of the curves, according to the asymptotic value $S_{100}(\infty)$.

\section{Quench from $h_{0}>1$ to $h>1$.}

Finally we consider the most general quench into the paramagnetic phase. Some numerical results for $\ell=100$ are shown in Fig. 6. Due to the double variation of the parameters, these figures are not easily readable. The most interesting features are:

- The crossover from the linear to non-linear regime is always at $t^{*} \simeq \ell / 2$.

- At fixed $h_{0}$, when the curves are rescaled according to $S_{\ell}(t=\infty)$, they do not fall on the same curve as for $h_{0}=\infty$.

- The dashed lines in Fig. 6 are the leading asymptotic results for large $\ell$ as given by Eq. (3.19). From the figures it is not always clear whether Eq. (3.19) is always smaller than the actual value (especially for small $h_{0}$ ). We checked in all the cases not enough clear graphically (i.e. $h_{0}=1.1$ with $h=10,5,2$ and $h_{0}=1.5$ with $\left.h=10,5\right)$ that for longer non-depicted times the curves finally cross the value given by Eq. (3.19). This shows quite firmly that for all $h, h_{0}$ the leading corrections to Eq. (3.19) are positive.

- The actual asymptotic values for $t \rightarrow \infty$ for the cases with $h$ and $h_{0}$ interchanged are close, but not exactly equal as in Eq. (3.19). We checked that, for larger $\ell$, their difference is almost constant and so can be imputed to finite (or $O(\log \ell)$ ) corrections that consequently have not the symmetry under the exchange of $h$ and $h_{0}$ as the leading term. 


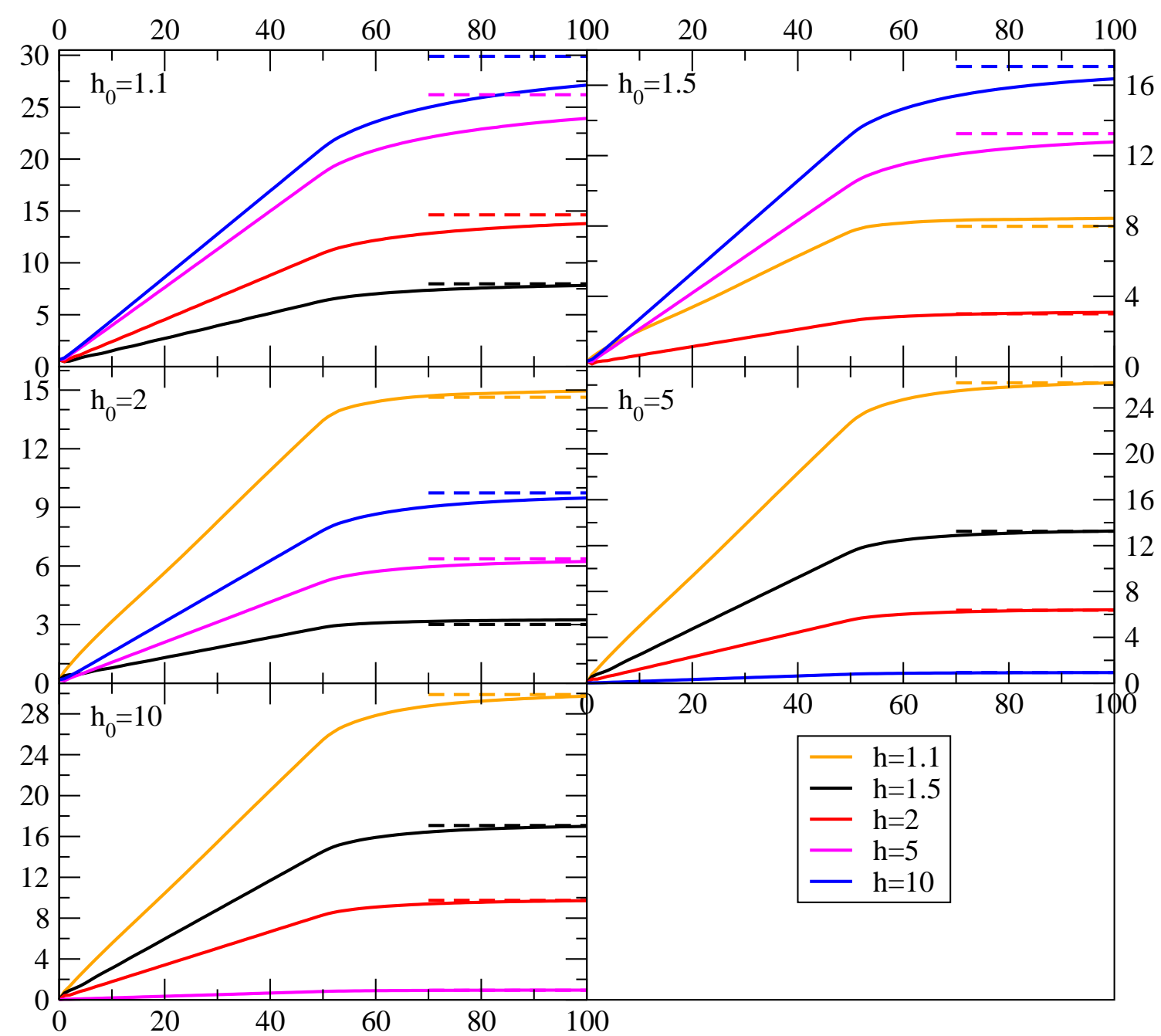

FIG. 6. $S_{100}(t)$ for a general quench from some generic $h_{0}$ to several $h$. The dashed lines are the leading asymptotic results for large $\ell$ Eq. (3.19).

\section{PHYSICAL INTERPRETATION}

The qualitative, and many of the quantitative, features of $S_{A}(t)$ found in the last two sections may be understood physically as follows. We emphasize that this is not an ab initio calculation but rather a simplified model which allows us to explain these observations. The initial state $\left|\psi_{0}\right\rangle$ has a very high energy relative to the ground state of the hamiltonian $H(\lambda)$ which governs the subsequent time evolution, and therefore acts as a source of quasiparticle excitations. Particles emitted from different points (further apart than the correlation length in the initial state) are incoherent, but pairs of particles moving to the left or right from a given point are highly entangled. We suppose that the cross-section for producing such a pair of particles of momenta $\left(p^{\prime}, p^{\prime \prime}\right)$ is $f\left(p^{\prime}, p^{\prime \prime}\right)$, and that, once they separate, they move classically. This will of course depends on $H(\lambda)$ and the state $\left|\psi_{0}\right\rangle$, and in principle is calculable, but we made no strong assumptions on its form. If the quasiparticle dispersion relation is $E=E(p)$, the classical velocity is $v(p)=d E / d p$. We assume that there is a maximum allowed speed which is taken to be 1 , that is $|v(p)| \leq 1$. A quasiparticle of 

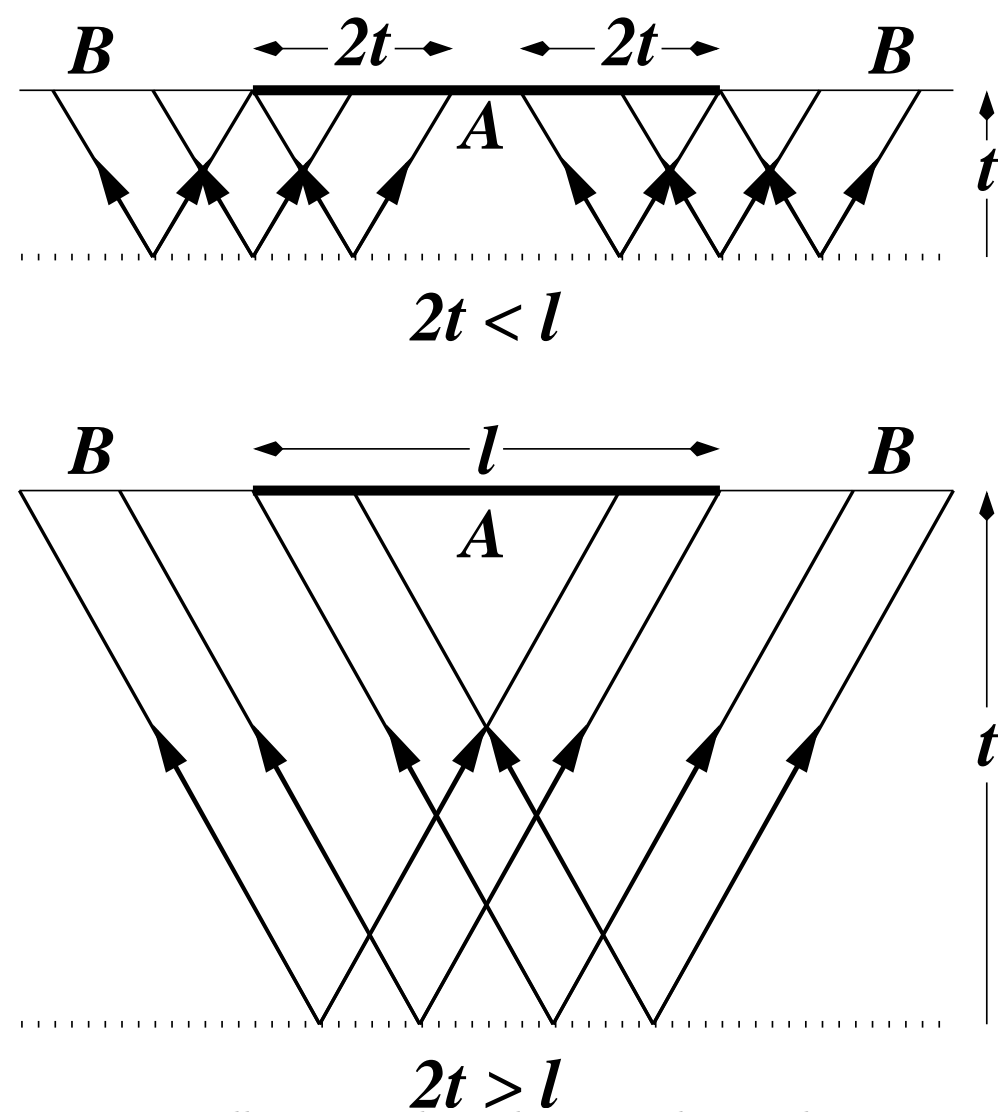

FIG. 7. Space-time picture illustrating how the entanglement between an interval A and the rest of the system, due to oppositely moving coherent quasiparticles, grows linearly and then saturates. The case where the particles move only along the light cones is shown here for clarity.

momentum $p$ produced at $x$ is therefore at $x+v(p) t$ at time $t$, ignoring scattering effects.

Now consider these quasiparticles as they reach either $A$ or $B$ at time $t$. The field at some point $x^{\prime} \in A$ will be entangled with that at a point $x^{\prime \prime} \in B$ if a pair of entangled particles emitted from a point $x$ arrive simultaneously at $x^{\prime}$ and $x^{\prime \prime}$ (see Fig. 7).

The entanglement entropy between $x^{\prime}$ and $x^{\prime \prime}$ is proportional to the length of the interval in $x$ for which this can be satisfied. Thus the total entanglement entropy is

$$
S_{A}(t) \approx \int_{x^{\prime} \in A} d x^{\prime} \int_{x^{\prime \prime} \in B} d x^{\prime \prime} \int_{-\infty}^{\infty} d x \int f\left(p^{\prime}, p^{\prime \prime}\right) d p^{\prime} d p^{\prime \prime} \delta\left(x^{\prime}-x-v\left(p^{\prime}\right) t\right) \delta\left(x^{\prime \prime}-x-v\left(p^{\prime \prime}\right) t\right) .
$$

Now specialize to the case where $A$ is an interval of length $\ell$. The total entanglement is twice that between $A$ and the real axis to the right of $A$, which corresponds to taking $p^{\prime}<0, p^{\prime \prime}>0$ in the above. The integrations over the coordinates then give $\max \left(\left(v\left(-p^{\prime}\right)+\right.\right.$ $\left.\left.v\left(p^{\prime \prime}\right)\right) t, \ell\right)$, so that

$$
\begin{aligned}
S_{A}(t) & \approx 2 t \int_{-\infty}^{0} d p^{\prime} \int_{0}^{\infty} d p^{\prime \prime} f\left(p^{\prime}, p^{\prime \prime}\right)\left(v\left(-p^{\prime}\right)+v\left(p^{\prime \prime}\right)\right) H\left(\ell-\left(v\left(-p^{\prime}\right)+v\left(p^{\prime \prime}\right)\right) t\right)+ \\
& +2 \ell \int_{-\infty}^{0} d p^{\prime} \int_{0}^{\infty} d p^{\prime \prime} f\left(p^{\prime}, p^{\prime \prime}\right) H\left(\left(v\left(-p^{\prime}\right)+v\left(p^{\prime \prime}\right)\right) t-\ell\right)
\end{aligned}
$$


where $H(x)=1$ if $x>0$ and zero otherwise. Now since $|v(p)| \leq 1$, the second term cannot contribute if $t<t^{*}=\ell / 2$, so that $S_{A}(t)$ is strictly proportional to $t$. On the other hand as $t \rightarrow \infty$, the first term is negligible (this assumes that $v(p)$ does not vanish except at isolated points), and $S_{A}$ is asymptotically proportional to $\ell$, as found earlier.

However, unless $|v|=1$ everywhere (as is the case for the conformal field theory calculation), $S_{A}$ is not strictly proportional to $\ell$ for $t>t^{*}$. In fact, it is easy to see that the asymptotic limit is always approached from below, as found for the Ising spin chain in Sec. III. The rate of approach depends on the behavior of $f\left(p^{\prime}, p^{\prime \prime}\right)$ in the regions where $v\left(-p^{\prime}\right)+v\left(p^{\prime \prime}\right) \rightarrow 0$. This generally happens at the zone boundary, and, for a non-critical

quench, also at $p^{\prime}=p^{\prime \prime}=0$. If we assume that $f$ is non-zero in those regions, we find a correction term $\sim-\ell^{3} / t^{2}$ in the limit where $t \gg t^{*}$.

The above result may be generalized to the case when A consists of several disjoint intervals, and checked against the corresponding conformal invariance result in the case where $|v(p)|=1$. This is discussed in Appendix C. In particular one should note that $S_{A}(t)$ is not always non-decreasing. For example, if A consists of a regular array of intervals, each of length $\ell$, separated by gaps also of length $\ell$, the entanglement entropy oscillates in a saw-tooth fashion with a period $\ell$.

\section{DISCUSSION}

We have presented results on the time-dependence of the entanglement entropy $S_{A}$, starting from a translationally invariant pure state $\left|\psi_{0}\right\rangle$ which is not an eigenstate of the time evolution operator, from two complementary perspectives. The first was conformal field theory, which applies only in the asymptotic regime, at the critical point, to conformally invariant initial states, in theories with a purely relativistic dispersion relation. In order to regularize these calculations and maintain tractability we were forced to apply a high energy cut-off $e^{-\epsilon E}$ to the state. The second was a solvable lattice model, the Ising model in a transverse field, in which we were able to perform calculations starting from a variety of initial states, considering both critical and non-critical quenches. In these calculations the only dimensional microscopic parameter is the lattice spacing. It should be noted that the continuum limit of the critical transverse Ising spin chain is known to correspond to a conformal field theory.

The results of these two sets of calculations have a number of important features in common: the entanglement entropy increases linearly with time $t$ (after transients die away in the lattice case), up to $t^{*}=\ell / 2$, in units where the maximum propagation speed of excitations is taken to be unity. For $t \gg t^{*}, S_{A} / \ell$ saturates at an asymptotic value.

However, there are also a number of differences. Partly these may be explained by the set-up being different: in the CFT calculation the scale of slope of the linear $t$-dependence, as well as the asymptotic value of $S_{A} / \ell$, is set by $\epsilon$, while for the lattice calculation it is set by the lattice spacing as well as the parameter $h_{0}$ specifying the initial state. However, in the CFT calculation the discontinuity in the slope is rounded only over a time interval $O(\epsilon)$, after which $S_{A}$ reaches its asymptotic value immediately. This means that the ratio

$$
R \equiv \frac{\left(\partial S_{A} / \partial t\right)_{t<t^{*}}}{2\left(\partial S_{A} / \partial \ell\right)_{t \gg t^{*}}}
$$


is exactly unity (in units where the maximum speed of propagation is 1.) On the other hand, the lattice calculations exhibit a slow increase towards the asymptotic value for $t>t^{*}$, so that $R$ is always less than unity. In Sec. IV however, we argue that this can be understood within a simple physical picture and the fact that, on the lattice, there are quasiparticle excitations which travel with a group velocity less than the maximum allowed value. This also accounts for the existence of a sharp feature in $S_{A}(t)$ even for non-critical quenches to $h>1$, which should correspond, in the scaling limit, to a relativistic theory with a mass gap. In these cases we find the explicit formula

$$
R=\frac{\int_{-\infty}^{0} d p^{\prime} \int_{0}^{\infty} d p^{\prime \prime} f\left(p^{\prime}, p^{\prime \prime}\right)\left[v\left(-p^{\prime}\right)+v\left(p^{\prime \prime}\right)\right]}{2 \int_{-\infty}^{0} d p^{\prime} \int_{0}^{\infty} d p^{\prime \prime} f\left(p^{\prime}, p^{\prime \prime}\right)} \leq 1
$$

There are however other puzzling features of the Ising results, which may however be specific to this model, for example the facts that the limiting behavior $\lim _{\ell \rightarrow \infty} S_{A} / \ell$ is a symmetric function of $h_{0}$ and $h$, and that the detailed time-dependences of all the quenches from $h_{0}=\infty$ to any value of $h>1$ all appear to collapse all on the same curve when suitably rescaled. Clearly these deserve more investigation to see whether they persist in other models.

In this paper, we have restricted attention to quenches from a translationally invariant initial state $\left|\psi_{0}\right\rangle$. It is also interesting to consider other setups, for example when initially $A$ and $B$ are completely decoupled and in their respective ground states. This is currently under investigation. It should also be noted that the causal picture of Sec. (IV) may be simply generalized to higher dimensions, with results which are, however, more dependent on the precise geometry.

Finally we should point out that the increase of the entanglement entropy we have found for a single interval $A$ in an infinite system is in no way connected with the second law of thermodynamics. As shown in Appendix C, in more complicated situations, it may decrease or even oscillate.

\section{ACKNOWLEDGMENTS}

We thank Rosario Fazio for bringing the problem of the dynamics of entanglement to our attention. PC thanks Fabian Essler and Alex Lefevre for useful discussions and comments about Toeplitz determinants. This work was supported in part by the EPSRC under Grant GR/R83712/01. Part of it was carried out while JC was a member of the Institute for Advanced Study. He thanks the School of Mathematics and the School of Natural Sciences for their hospitality. This stay was supported by the Ellentuck Fund.

\section{APPENDIX A: COMPUTATION OF $S_{\ell}$ FOR THE ISING CHAIN}

The diagonalization of $H_{I}$ is presented in several standard textbooks (see e.g. [16]). Nevertheless we briefly present it here to make the notations self-explanatory. The hamiltonian 
for a chain of length $N$ is

$$
H_{I}=-\frac{1}{2} \sum_{l=-(N-1) / 2}^{(N-1) / 2}\left[\sigma_{l}^{x} \sigma_{l+1}^{x}+h \sigma_{l}^{z}\right]
$$

and we consider open boundary conditions. We are interested in the limit $N \rightarrow \infty$. The first step in diagonalizing the hamiltonian is to introduce a set of spinless fermion annihilation and creation operators through the non-local Jordan-Wigner transformations

$$
\hat{a}_{l}=\left(\prod_{m<l} \sigma_{m}^{z}\right) \frac{\sigma_{l}^{x}-i \sigma_{l}^{y}}{2}, \quad \hat{a}_{l}^{\dagger}=\left(\prod_{m<l} \sigma_{m}^{z}\right) \frac{\sigma_{l}^{x}+i \sigma_{l}^{y}}{2},
$$

where $\hat{a}_{l}^{\dagger}$ stands for the Hermitian conjugate of $\hat{a}_{l}$. They satisfy the anticommutation relations

$$
\left\{\hat{a}_{l}^{\dagger}, \hat{a}_{m}\right\}=\delta_{l m}, \quad\left\{\hat{a}_{l}, \hat{a}_{m}\right\}=0 .
$$

Let us now introduce the Fourier transformed fermionic operators

$$
\hat{d}_{k}=\frac{1}{\sqrt{N}} \sum_{l=-(N-1) / 2}^{(N-1) / 2} \hat{a}_{l} e^{-i \frac{2 \pi}{N} k l}
$$

with $-(N-1) / 2 \leq k \leq(N-1) / 2$. Since this transformation is unitary, the anticommutation relations $\left\{\hat{d}_{k}^{\dagger}, \hat{d}_{p}\right\}=\delta_{k p}$ remain valid. The hamiltonian now takes the form

$$
H_{I}=\sum_{k=-(N-1) / 2}^{(N-1) / 2}\left(\left[h-\cos \frac{2 \pi k}{N}\right] \hat{d}_{k}^{\dagger} \hat{d}_{k}-\frac{i}{2} \sin \frac{2 \pi k}{N}\left[\hat{d}_{-k} \hat{d}_{k}+\hat{d}_{-k}^{\dagger} \hat{d}_{k}^{\dagger}\right]-\frac{h}{2}\right) .
$$

A final unitary transformation is needed to cast the hamiltonian into diagonal form. This is the so-called Bogoliubov transformation

$$
\hat{b}_{k}^{\dagger}=u_{k} \hat{d}_{k}^{\dagger}+i v_{k} \hat{d}_{-k}, \quad \hat{b}_{k}=u_{k} \hat{d}_{k}-i v_{k} \hat{d}_{-k}^{\dagger},
$$

where $u_{k}=\cos \theta_{k} / 2, v_{k}=\sin \theta_{k} / 2$ and

$$
\tan \theta_{k}=\frac{\sin \frac{2 \pi k}{N}}{\cos \frac{2 \pi}{N} k-h}
$$

Again, due to unitarity of the Bogoliubov transformation, the operators $\left\{\hat{b}_{k}\right\}$ follow the usual anticommutation relations $\left\{\hat{b}_{k}^{\dagger}, \hat{b}_{p}\right\}=\delta_{k p}$. Finally, the hamiltonian takes a diagonal form

$$
H_{I}=\sum_{k=-(N-1) / 2}^{(N-1) / 2} \epsilon_{k}\left(\hat{b}_{k}^{\dagger} \hat{b}_{k}-1 / 2\right),
$$

where

$$
\epsilon_{k} \equiv \sqrt{\left(h-\cos \frac{2 \pi k}{N}\right)^{2}+\sin ^{2} \frac{2 \pi k}{N}}
$$


The thermodynamic limit is obtained by defining $\varphi=2 \pi k / N$ and sending $N \rightarrow \infty$, giving

$$
H_{I}=\int_{-\pi}^{\pi} \frac{\mathrm{d} \varphi}{2 \pi} \epsilon_{\varphi} \hat{b}_{\varphi}^{\dagger} \hat{b}_{\varphi}
$$

with

$$
\epsilon_{\varphi}^{2}=(h-\cos \varphi)^{2}+\sin ^{2} \varphi,
$$

where we omit a constant term from the hamiltonian.

For the time evolution, we closely follow Ref. [15], whose results are mainly based on some previous works $[18,19]$. Since the hamiltonian is translational invariant, only fermionic states with opposite momentum $k$ and $-k$ are mixed. We may therefore write the two component column vectors

$$
\hat{B}_{k}=\left(\begin{array}{c}
\hat{b}_{k} \\
\hat{b}_{-k}^{\dagger}
\end{array}\right), \quad \text { and } \quad \hat{\mathrm{D}}_{\mathrm{k}}=\left(\begin{array}{c}
\hat{d}_{k} \\
\hat{d}_{-k}^{\dagger}
\end{array}\right) .
$$

The Bogoliubov transformation relating $\hat{B}_{k}$ and $\hat{D}_{k}$ is expressed as $\hat{D}_{k}=R_{x}\left(\theta_{k}\right) \hat{B}_{k}$, where

$$
R_{\mu}(\alpha)=\cos \frac{\alpha}{2}+i \sigma_{\mu} \sin \frac{\alpha}{2}
$$

and $\sigma_{\mu}$ are the Pauli matrices with $\mu=x, y, z$. (These are used for conciseness of notation and should not be confused with the operators representing the 'spins' of the Ising chain.)

For $t<0$, the system is taken to be in the ground state corresponding to the field $h_{0}$. It is natural to define $\hat{b}^{\prime}$ fermions as those which diagonalize the hamiltonian with $h_{0}$. Similarly, $\theta_{k}^{\prime}$ and $\hat{B}_{k}^{\prime}$ are given by analogy with (A7) and (A6) with $h$ replaced by $h_{0}$. The state $\left|\psi_{0}\right\rangle$ is therefore the vacuum of $\hat{B}^{\prime}$ fermions. This in particular implies

$$
\left\langle\psi_{0}\left|B_{k}^{\prime} B_{k}^{\prime \dagger}\right| \psi_{0}\right\rangle=\left(\begin{array}{ll}
1 & 0 \\
0 & 0
\end{array}\right)=\frac{1}{2}\left(\sigma^{z}+1\right),
$$

that in terms of $B_{k}$ fermions reads

$$
\left\langle\psi_{0}\left|B_{k} B_{k}^{\dagger}\right| \psi_{0}\right\rangle=R_{x}\left(\theta_{k}^{\prime}-\theta_{k}\right) \frac{1}{2}\left(\sigma^{z}+1\right) R_{x}^{\dagger}\left(\theta_{k}^{\prime}-\theta_{k}\right)=\frac{1}{2}\left(1+\sigma^{z} \cos \phi_{k}+\sigma^{y} \sin \phi_{k}\right),
$$

with $\phi_{k}=\theta_{k}^{\prime}-\theta_{k}$.

The time evolution of the operators now proceeds according to the hamiltonian (A8), so that $B_{k}(t)=U_{k}(t) B_{k}(0)$, where

$$
U_{k}(t) \equiv e^{-i H_{I} t}=\left(\begin{array}{cc}
e^{-i \epsilon_{k} t} & 0 \\
0 & e^{i \epsilon_{k} t}
\end{array}\right)=R_{z}\left(-2 \epsilon_{k} t\right) .
$$

The expectation values at any time can be easily evaluated. Let us introduce the operators

$$
\omega_{i}^{+}=\hat{a}_{i}^{\dagger}+\hat{a}_{i}, \quad \text { and } \quad \omega_{i}^{-}=\hat{a}_{i}^{\dagger}-\hat{a}_{i},
$$

and the two-component vector operator

$$
\Omega_{k}(t)=\left(\begin{array}{c}
\omega_{k}^{+}(t) \\
\omega_{k}^{-}(t)
\end{array}\right)=\sqrt{2} R_{y}\left(\frac{\pi}{2}\right) \hat{D}_{k}(t),
$$


where we defined $\omega_{k}^{ \pm}$as the Fourier transform of $\omega_{i}^{ \pm}$. Using the algebra of $S U(2)$ matrices, it is straightforward to find [15]

$$
\begin{aligned}
\left\langle\psi_{0}\left|\Omega_{k} \Omega_{k}^{\dagger}\right| \psi_{0}\right\rangle & =\left\langle\psi_{0}\left|\left(\begin{array}{cc}
\omega_{k}^{+} \omega_{-k}^{+} & -\omega_{k}^{+} \omega_{-k}^{-} \\
\omega_{k}^{-} \omega_{-k}^{+} & -\omega_{k}^{-} \omega_{-k}^{-}
\end{array}\right)\right| \psi_{0}\right\rangle= \\
& =\left(\begin{array}{cc}
1-\sin \phi_{k} \sin 2 \epsilon_{k} t & -e^{i \theta_{k}}\left(\cos \phi_{k}+i \sin \phi_{k} \cos 2 \epsilon_{k} t\right) \\
-e^{-i \theta_{k}}\left(\cos \phi_{k}-i \sin \phi_{k} \cos 2 \epsilon_{k} t\right) & 1+\sin \phi_{k} \sin 2 \epsilon_{k} t
\end{array}\right) .
\end{aligned}
$$

Transforming back to real space we have

$$
\begin{aligned}
& \left\langle\omega_{l}^{+} \omega_{j}^{+}\right\rangle=\frac{1}{N} \sum_{k} e^{i k(l-j)}\left(1-\sin \phi_{k} \sin 2 \epsilon_{k} t\right), \\
& \left\langle\omega_{l}^{-} \omega_{j}^{-}\right\rangle=\frac{1}{N} \sum_{k} e^{i k(l-j)}\left(-1-\sin \phi_{k} \sin 2 \epsilon_{k} t\right), \\
& \left\langle\omega_{l}^{-} \omega_{j}^{+}\right\rangle=\frac{1}{N} \sum_{k} e^{i k(l-j)} e^{-i \theta_{k}}\left(-\cos \phi_{k}+i \sin \phi_{k} \cos 2 \epsilon_{k} t\right) .
\end{aligned}
$$

Taking the thermodynamic limit, the sum over $k$ becomes an integral over $\varphi$ and

$$
\begin{aligned}
\tan \theta_{k} & \rightarrow \tan \theta_{\varphi}=\frac{\sin \varphi}{\cos \varphi-h} \\
\phi_{k} & \rightarrow \Phi_{\varphi}=\theta_{\varphi}^{\prime}-\theta_{\varphi}
\end{aligned}
$$

and $\epsilon_{k} \rightarrow \epsilon_{\varphi}$ defined by Equation (A11).

As stated in the text, it is useful to introduce two Majorana operators [2] at each site of the spin chain according to equation (3.2). Operators $\check{a}_{m}$ are Hermitian and obey anticommutation relations,

$$
\check{a}_{m}^{\dagger}=\check{a}_{m}, \quad\left\{\check{a}_{m}, \check{a}_{n}\right\}=2 \delta_{m n} .
$$

In terms of the Dirac operators introduced by the Jordan-Wigner transformation they read

$$
\begin{array}{cc}
\check{a}_{2 l-1}=\hat{a}_{l}^{\dagger}+\hat{a}_{l}, & \check{a}_{2 l}=-i\left(\hat{a}_{l}^{\dagger}-\hat{a}_{l}\right), \\
\hat{a}_{l}=\frac{\check{a}_{2 l-1}-i \check{a}_{2 l}}{2}, & \hat{a}_{l}^{\dagger}=\frac{\check{a}_{2 l-1}+i \check{a}_{2 l}}{2},
\end{array}
$$

and so, in terms of $\omega^{ \pm}$they are

$$
\check{a}_{2 l-1}=\omega_{l}^{+}, \quad \check{a}_{2 l}=-i \omega_{l}^{-} .
$$

The two point time-dependent expectation values of $\check{a}$ are easily obtained in terms of Eqs. (A20) as

$$
\begin{aligned}
\left\langle\check{a}_{2 l} \check{a}_{2 m}\right\rangle & =-\left\langle\omega_{l}^{-} \omega_{m}^{-}\right\rangle, \\
\left\langle\check{a}_{2 l-1} \check{a}_{2 m-1}\right\rangle & =\left\langle\omega_{l}^{+} \omega_{m}^{+}\right\rangle, \\
\left\langle\check{a}_{2 l} \check{a}_{2 m-1}\right\rangle & =-i\left\langle\omega_{l}^{-} \omega_{m}^{+}\right\rangle, \\
\left\langle\check{a}_{2 l-1} \check{a}_{2 m}\right\rangle & =-i\left\langle\omega_{l}^{+} \omega_{m}^{-}\right\rangle .
\end{aligned}
$$


The entanglement entropy for the time-dependent state is now obtained exactly as in the ground-state, following closely Ref. [2]. From equation (A25), the time dependent correlation matrix $\left\langle\check{a}_{m} \check{a}_{n}\right\rangle=\delta_{m n}+i \Gamma_{m n}^{A}$ of the Majorana operators $\check{a}$ reads

$$
\Gamma^{A}=\left[\begin{array}{cccc}
\Pi_{0} & \Pi_{-1} & \cdots & \Pi_{1-N} \\
\Pi_{1} & \Pi_{0} & & \vdots \\
\vdots & & \ddots & \vdots \\
\Pi_{N-1} & \cdots & \cdots & \Pi_{0}
\end{array}\right], \quad \Pi_{l}=\left[\begin{array}{cc}
-f_{l} & g_{l} \\
-g_{-l} & f_{l}
\end{array}\right]
$$

The coefficients $g_{l}$ and $f_{l}$, in the limit of an infinite chain are easily derived from equations (A20) and (A25), obtaining the results reported in the text equation (3.4).

As shown in Ref. [2], the entropy of the reduced density matrix $\rho$ for $\ell$ adjacent spins can be computed from $\Gamma^{A}$, in spite of the non-local character of transformation (3.2). Indeed, the symmetry

$$
\left(\prod_{l} \sigma_{l}^{z}\right) H_{I}\left(\prod_{l} \sigma_{l}^{z}\right)=H_{I}
$$

implies that the trace over a given spin at the site $j$ is equivalent to the trace over the two Majorana operators at the same site. Thus the density matrix $\rho_{\ell}$ can be reconstructed from the restricted $2 \ell \times 2 \ell$ correlation matrix

$$
\left\langle\check{a}_{m} \check{a}_{n}\right\rangle=\delta_{m n}+i\left(\Gamma_{\ell}^{A}\right)_{m n}, \quad m, n=1, \cdots, 2 \ell,
$$

where $\Gamma_{\ell}^{A}$ is the matrix obtained by $\Gamma^{A}$ cutting the last $N-\ell$ rows and columns.

An orthogonal matrix $V$ bring $\Gamma_{\ell}^{A}$ into the block-diagonal form

$$
V \Gamma_{\ell}^{A} V^{T}=\bigoplus_{l=1}^{\ell} \nu_{l}\left[\begin{array}{cc}
0 & 1 \\
-1 & 0
\end{array}\right]
$$

This means that the reduced density matrix can be written as a product $\rho_{\ell}=\varrho_{1} \otimes \cdots \otimes \varrho_{\ell}$, where each $\varrho_{l}$ has eigenvalues $\left(1 \pm \nu_{l}\right) / 2$ and entropy $S\left(\varrho_{l}\right)=H\left(\nu_{l}\right)$, where $H(x)$ is given by equation (3.6). Thus the entropy of $\rho_{\ell}$ is the sum of entropies of the $\ell$ uncorrelated modes,

$$
S_{\ell}=\sum_{l=1}^{\ell} H\left(\nu_{l}\right)
$$

Summarizing: for arbitrary time, and in the thermodynamic limit $(N \rightarrow \infty)$, the time evolution of the entropy $S_{\ell}$ of the Ising model can be obtained by

- evaluating Eq. (3.4) numerically for $l=0, \cdots, \ell-1$,

- diagonalizing $\Gamma_{\ell}^{A}$ to obtain $\nu_{l}$, and

- evaluating $S_{\ell}$ using Eq. (A30). 


\section{APPENDIX B: SOME ANALYTIC RESULTS FOR THE CORRELATION MATRIX}

In some cases it is possible to get analytic expression for the coefficients $g_{l}$ and $f_{l}$, that help the numerical computation in the general case. For example, in the limit $h_{0} \rightarrow \infty$ and for $t \rightarrow \infty$ we get

$$
g_{l}=\frac{1}{2 \pi} \int_{0}^{2 \pi} d \varphi e^{-i \varphi l} \frac{\left(e^{-i \varphi}-h\right)}{\epsilon_{\varphi}^{2}}(h-\cos \varphi)=\left\{\begin{array}{l}
\frac{1-h^{2}}{2 h^{l+2}} \quad l \geq 1 \\
\frac{1}{2 h^{2}}-1 \quad l=0 \\
\frac{1}{2 h} \quad l=-1 \\
0 \quad l \leq-2
\end{array}\right.
$$

Using these values in equation (3.4) reduces the difficulty of the numerical calculations for generic times.

For the a critical quench $\left(h_{0}=\infty\right.$ and $\left.h=1\right)$, the infinite time value of $g_{l}$ reduces to

$$
g_{l}=\frac{1}{4 \pi} \int_{0}^{2 \pi} d \varphi\left[e^{-i \varphi(l+1)}-e^{-i \varphi l}\right]=\frac{\delta_{l,-1}-\delta_{l, 0}}{2}
$$

In this case, the Toeplitz matrix has only the two diagonals closest to the principal with non-zero entries $\pm 1 / 2$. This matrix is nothing but the Laplacian on the lattice (minus 1 , and without two elements at $(2 \ell, 1)$ and $(1,2 \ell)$ that for $\ell \rightarrow \infty$ are irrelevant). Thus the eigenvalues for large $\ell$ are

$$
\nu_{m}=\cos \frac{\pi m}{2 \ell} \quad m=1, \ldots \ell
$$

and the entropy is

$$
\lim _{\ell \rightarrow \infty} \frac{S_{\ell}(\infty)}{\ell}=\frac{1}{\ell} \sum_{m=1}^{\ell} H\left(\cos \frac{\pi m}{2 \ell}\right) \rightarrow \frac{2}{\pi} \int_{0}^{\pi} d y H(\cos y)=2\left(\log 2-\frac{1}{2}\right)
$$

\section{APPENDIX C: GENERAL RESULT FOR AN ARBITRARY NUMBER OF INTERVALS.}

In this appendix we sketch the CFT calculation of $S_{A}(t)$ for the case when $A$ consists of the union of the $N$ intervals $\left(u_{2 j-1}, u_{2 j}\right)$ where $1 \leq j \leq N$ and $u_{k}<u_{k+1}$. $\operatorname{Tr} \rho_{A}^{n}$ is given as usual by the ratio $Z_{n} / Z_{1}^{n}$ which has the form of a correlation function

$$
\left\langle\prod_{j} \Phi_{n}\left(u_{2 j-1}+i \tau_{1}\right) \prod_{j} \Phi_{-n}\left(u_{2 j}+i \tau_{1}\right)\right\rangle,
$$

in a strip of width $2 \epsilon$. This is related to the upper half $z$-plane by $w=(2 \epsilon / \pi) \log z$, whereby the points $u_{k}+i \tau_{1}$ are mapped to $z_{k}=e^{\pi\left(u_{k}+i \tau_{1}\right) / 2 \epsilon}$. If we choose $\sum_{k} u_{k}=0$, the jacobian factors in the transformation are constant, independent of the $u_{k}$. Analytically continuing to $\tau_{1}=\epsilon+i t$ sets $z_{k}=i e^{\pi\left(u_{k}-t\right) / 2 \epsilon}$, that is, on the imaginary axis, while the image points go over into $\bar{z}_{k}=-i e^{\pi\left(u_{k}+t\right) / 2 \epsilon}$. The correlation function (C1) is a generalization of that in (2.5) 
and is a product of several factors: the first involves differences $\left(z_{k}-z_{l}\right)$ raised to the power $\pm 2 n \Delta_{n}$ according to whether $k-l$ is even or odd; the second is the same with $z_{k}$ replaced by $\bar{z}_{k}$; and the third involves factors like $\left(z_{k}-\bar{z}_{l}\right)$. It can be seen that the $t$-dependence cancels between the first and second type of terms. On the other hand

$$
\left|z_{k}-\bar{z}_{l}\right|=e^{\pi\left(u_{k}-t\right) / 2 \epsilon}+e^{\pi\left(u_{l}+t\right) / 2 \epsilon} \sim e^{\pi \max \left(u_{k}-t, u_{l}+t\right) / 2 \epsilon}
$$

where we have assumed that $u_{k} \pm t \gg \epsilon$. This expression is to be raised to the power $\pm 2 \Delta_{n}$ according to whether $k-l$ is odd or even. Finally differentiating with respect to $n$ and setting $n=1$ we find

$$
S_{A}(t) \sim S_{A}(\infty)+\frac{\pi c}{12 \epsilon} \sum_{k, l}(-1)^{k-l-1} \max \left(u_{k}-t, u_{l}+t\right) .
$$

If $N$ is finite (or more generally the $u_{k}$ are bounded) the second term vanishes for sufficiently large $t$. At shorter times, $S_{A}(t)$ exhibits piecewise linear behavior in $t$ with cusps whenever $2 t=u_{k}-u_{l}$, at which the slope changes by $\pm(\pi c / 6 \epsilon$ according to whether $k-l$ is even or odd. In the case of an infinite number of regular intervals, with $u_{k}=k \ell, k \in \mathrm{Z}, S_{A}(t)$ exhibits a sawtooth behavior. 


\section{REFERENCES}

[1] A. Osterloh, L. Amico, G. Falci and R. Fazio, Scaling of Entanglement close to a Quantum Phase Transitions, 2002 Nature 416608 [quant-ph/0202029]

T. J. Osborne and M. A. Nielsen, Entanglement in a simple quantum phase transition, 2002 Phys. Rev. A 6632110 [quant-ph/0202162]

H. Barnum, E. Knill, G. Ortiz, R. Somma, and L. Viola, A Subsystem-Independent Generalization of Entanglement, 2004 Phys. Rev. Lett. 92107902 [quant-ph/0305023]

F. Verstraete, M. A. Martin-Delgado, and J. I. Cirac, Diverging Entanglement Length in Gapped Quantum Spin Systems, 2004 Phys. Rev. Lett. 92087201 [quant-ph/0311087] T.-C.Wei, D. Das, S. Mukhopadyay, S. Vishveshwara, and P. M. Goldbart, Global entanglement and quantum criticality in spin chains, 2004 quant-ph/0405162

G. Toth, Entanglement Witnesses in Spin Models, 2004 Phys. Rev. A $71010301(\mathrm{R})$ [quant-ph/0406061]

M. Popp, F. Verstraete, M. A. Martin-Delgado, and J. I. Cirac, Localizable Entanglement, 2004 quant-ph/0411123

[2] G. Vidal, J. I. Latorre, E. Rico, and A. Kitaev, Entanglement in quantum critical phenomena, 2003 Phys. Rev. Lett. 90227902 [quant-ph/0211074]

J. I. Latorre, E. Rico, and G. Vidal, Ground state entanglement in quantum spin chains, 2004 Quant. Inf. and Comp. 4048 [quant-ph/0304098]

[3] P. Calabrese and J. Cardy, Entanglement entropy and quantum field theory, 2004 JSTAT P06002 [hep-th/0405152]

[4] C. Holzhey, F. Larsen, and F. Wilczek, Geometric and Renormalized Entropy in Conformal Field Theory, 1994 Nucl. Phys. B 42444 [hep-th/9403108]

[5] M. Srednicki, Entropy and Area, 1993 Phys. Rev. Lett. 71666 [hep-th/9303048]

[6] B.-Q. Jin and V. E. Korepin, Quantum Spin Chain, Toeplitz Determinants and FisherHartwig Conjecture, 2004 J. Stat. Phys. 11679 [quant-ph/0304108]

[7] A. R. Its, B.-Q. Jin and V. E. Korepin, 2005 Entanglement in XY Spin Chain Preprint quant-ph/0409027

[8] I. Peschel, On the entanglement entropy for a XY spin chain, 2004 JSTAT P12005 [cond-mat/0410416]

[9] N. Lambert, C. Emary, and T. Brandes, Entanglement and the Phase Transition in Single Mode Super-radiance, 2004 Phys. Rev. Lett. 92073602 [quant-ph/0309027]

C. Wellard and R. Orus, Quantum phase transitions in antiferromagnetic planar cubic lattices, 2004 Phys. Rev. A 70062318 [quant-ph/0401144]

V. Popkov and M. Salerno, Logarithmic divergence of the block entanglement entropy for the ferromagnetic Heisenberg model, 2005 Phys. Rev. A 71012301 [quant-ph/0404026] M.B. Plenio, J. Eisert, J. Dreissig, and M. Cramer, Entropy, entanglement, and area: analytical results for harmonic lattice systems, 2005 Phys. Rev. Lett. 94060503 [quantph/0405142]

V. E. Korepin, Universality of Entropy Scaling in 1D Gap-less Models, 2004 Phys. Rev. Lett. 92096402 [cond-mat/0311056]

G. Refael and J. E. Moore, Entanglement entropy of random quantum critical points in one dimension, 2004 Phys. Rev. Lett. 93260602 [cond-mat/0406737]

J. P. Keating and F. Mezzadri, Random Matrix Theory and Entanglement in Quantum 
Spin Chains, 2004 Commun. Math. Phys. 252543 [quant-ph/0407047]

J. P. Keating and F. Mezzadri, Entanglement in Quantum Spin Chains, Symmetry Classes of Random Matrices, and Conformal Field Theory, 2005 Phys. Rev. Lett. 94 050501

H. Fan, V. Korepin, and V. Roychowdhury, Entanglement in a Valence-Bond-Solid State, 2004 Phys. Rev. Lett. 93227203 [quant-ph/0406067]

Y. Chen, P. Zanardi, Z. D. Wang, and F. C. Zhang, Entanglement and Quantum Phase Transition in Low Dimensional Spin Systems, 2004 quant-ph/0407228

G. Levine, Entanglement entropy in a boundary impurity model, 2004 Phys. Rev. Lett. 93266402 [cond-mat/0408366]

J. I. Latorre, R. Orus, E. Rico, and J. Vidal, Entanglement entropy in the LipkinMeshkov-Glick model, 2004 cond-mat/0409611

A. Hamma, R. Ionicioiu, and P. Zanardi, Bipartite entanglement and entropic boundary law in lattice spin systems, 2005 Phys. Rev. A 71022315 [quant-ph/0409073]

C. Mejía-Monasterio, G. Benenti, G. G. Carlo, and G. Casati, Entanglement Across a Transition to Quantum Chaos, quant-ph/0410246

V. Eisler and Z. Zimboras, Entanglement in the XX Spin Chain with Energy Current, 2004 quant-ph/0412118

I. Peschel, Entanglement entropy with interface defects, 2005 cond-mat/0502034

S. O. Skrovseth and K. Olaussen, Use of Entanglement to Identify Critical Systems, 2005 cond-mat/0503235

[10] W. Dür, L. Hartmann, M. Hein, and H. J. Briegel, Entanglement in spin chains and lattices with long-range interactions, 2005 Phys. Rev. Lett. 94097203 [quant-ph/0407075] J. Calsamiglia, L. Hartmann, W. Dür, and H. J. Briegel, Entanglement and decoherence in spin gases, 2005 quant-ph/0502017

[11] A. B. Zamolodchikov, Irreversibility of the flux of the renormalization group in a 2D field theory, 1986 JETP Lett. 43731 [1986 Pisma Zh. Eksp. Teor. Fiz. 43 565].

[12] J. I. Latorre, C. A. Lutken, E. Rico, and G. Vidal, Fine-grained entanglement loss along renormalization group flows, 2005 Phys. Rev. A 71034301 [quant-ph/0404120]

R. Orus, Entanglement and majorization in (1+1)-dimensional quantum systems, 2005 quant-ph/0501110

See also: J. Preskill, Quantum information and physics: some future directions, $2000 \mathrm{~J}$. Mod. Opt. 47127

T. M. Fiola, J. Preskill, A. Strominger, and S. P. Trivedi, Black hole thermodynamics and information loss in two dimensions, 1994 Phys. Rev. D 503987 [hep-th/9403137]

[13] L. Amico, A. Osterloh, F. Plastina, R. Fazio, and G. M. Palma. Dynamics of Entanglement in One-Dimensional Spin Systems, 2004 Phys. Rev. A 69022304 [quantph/0307048]

S. Montagero G. Bonenti, and R. Fazio, Dynamics of entanglement in quantum computers with imperfections, 2003 Phys. Rev. Lett. 91187901 [quant-ph/0307036]

J. Vidal, G. Palacios, and C. Aslangul, Entanglement dynamics in the Lipkin-MeshkovGlick model, 2004 Phys. Rev. A 70062304 [cond-mat/0406481]

A. Sen De, U. Sen, and M. Lewenstein, Nonergodicity of entanglement and its complementary behavior to magnetization in infinite spin chain, 2004 Phys. Rev. A 70 060304(R) [quant-ph/0407114] 
J. Wang, H. Batelaan, J. Podany, and A. F. Starace, Entanglement Evolution in the presence of decoherence, quant-ph/0503116

[14] M. Greiner, O. Mandel, T. Esslinger, T. W. Hänsch, and I. Bloch, Quantum phase transition from a superfluid to a Mott insulator in a gas of ultracold atoms, 2002 Nature (London) 41539

C. Orzel, A. K. Tuchman, M. L. Fenselau, M. Yasuda, and M. A. Kasevich, Squeezed States in a Bose-Einstein Condensate, 2001 Science 2912386

M. P. A. Fisher, P. B. Weichman, G. Grinstein, D. S. Fisher, Boson localization and the superfluid-insulator transition, 1989 Phys. Rev. B 40546

[15] K. Sengupta, S. Powell, and S. Sachdev, Quench dynamics across quantum critical points, 2004 Phys. Rev. A 69053616 [cond-mat/0311355]

[16] S. Sachdev, Quantum Phase Transitions, Cambridge Univ. Press (1999)

[17] H. Au-Yang and B. McCoy, Theory of layered Ising models. II. Spin correlation functions parallel to the layering, 1974 Phys. Rev. B 103885

[18] E. Barouch, B. McCoy, and M. Dresden, Statistical Mechanics of the XY Model. I, 1970 Phys. Rev. A 21075

E. Barouch and B. McCoy, Statistical Mechanics of the X Y Model. II. Spin-Correlation Functions, 1971 Phys. Rev. A 3786

E. Barouch and B. McCoy, Statistical Mechanics of the XY Model. III, 1971 Phys. Rev. A 32137

[19] F. Igloi and H. Rieger, Long-Range Correlations in the Nonequilibrium Quantum Relaxation of a Spin Chain, 2000 Phys. Rev. Lett. 853233 [cond-mat/0003193] 